Medical Center, Bronx, New York. Epilepsy occurred in $14 \%$ of autistic children and $8 \%$ of dysphasic children. Girls were affected more frequently than boys ( $24 \%$ cf $11 \%)$. The seizure types included generalized tonic-clonic, atypical absence, atonic, myoclonic, infantile spasms and partial. Infantile spasms occurred in $12 \%$ of autistic patients with epilepsy. The major risk factors for epilepsy were severe mental deficiency and the combination of mental deficiency with a motor deficit. When cognitive and motor disabilities were excluded, the risk of epilepsy in autistic children was only $6 \%$ and similar to that found in dysphasic non-autistic children (Tuchman RF, Rapin I, Shinnar S. Autistic and dysphasic children. II: epilepsy. Pediatrics Dec 1991; 88:1219-1225.) (Reprints: Dr. Rapin, Rose F. Kennedy Center for Research in Mental Retardation, Room 807, 1410 Pelham Parkway South, Bronx, NY 10461.)

COMMENT. The rates of epilepsy reported in autistic children have ranged from $11 \%$ to $42 \%$. Associated cognitive and motor deficits, a higher ratio of girls, and the complication of verbal auditory agnosia in some series might explain the higher rates of epilepsy. As a group, autistic children are more likely than dysphasic children to have language subtypes affecting central processing and a history of regression of language and behavior. Girls with autism are more likely than boys to have severe mental deficiency and a motor deficit.

Epilepsy occurred in $8(17 \%)$ of 46 children with autism and deafness (Jure $R$ et al. Hearing impaired autistic children. Dev Med Child Neurol Dec 1991; $\underline{33}: 1062-1072$ ).

\title{
SURGERY OF EPILEPSY
}

The surgical outcome in 34 patients between 2 and 15 years of age who were operated on for medically intractable seizures is reported from the Neurosurgical Clinic, University Clinical Center, Visegradska 26, Belgrade, Yugoslavia. Temporal epileptic foci were present in 9 patients, extratemporal foci in 6, infantile hemiplegia in 16 and epilepsia partialis continua in 3 . Detectable brain lesions were present in 30 (88\%). At 1-14 years postoperative follow-up (mean, 4 years), $21(62 \%)$ are seizure free, $8(23 \%)$ have improved, and $5(15 \%)$ have shown no improvement. Patients with temporal foci had a partial temporal lobectomy or restricted temporofrontal resection; those with extratemporal foci had cortical resection; and patients with infantile hemiplegia had a partial hemispherectomy, extensive cortical resection or functional hemispherectomy of Rasmussen type. (Ribaric II et al. Surgical treatment of epilepsy: our experiences with 34 children. Child's Nerv Syst Nov 1991; 7:402-404.) (Reprints: Dr. Ribaric, University Clinical Center, Visegradska 26, YU-11000 Belgrade, Yugoslavia.)

COMMENT. Of 16 patients with infantile hemiplegia who were subjected to hemispherectomy, $12(75 \%)$ were seizure free. Lindsay J et al. from the Park Hospital for Children, Oxford, found a similar incidence of seizure control following hemispherectomy in 17 children with hemiplegic epilepsy (Dev Med Child Neurol 1987; 29:592. See 
Surgery and Epilepsy in Progress in Pediatric Neurology, JG Millichap ed, 1991, pp 131-138).

\section{POST-OPERATIVE EPILEPSY RECURRENCE}

Post-operative seizures occurred in 72 patients after resective procedures for epilepsy at The Epilepsy Surgery Program, Departments of Neurology and Neurological Surgery, The Cleveland Clinic Foundation, Cleveland, $\mathrm{OH}$. The patients ranged in age from 2-47 years (mean 26 years). Temporal lobe resection had been performed in $74 \%$ and extratemporal resections in $26 \%$. The post-operative seizures were similar to preoperative patterns in $74 \% .86 \%$ of patients had their first seizure occurrence within 1 year of surgery. Of 21 patients with recurrences in the first week after operation, none had subtherapeutic antiepileptic drug levels. Those with first recurrences from 1 week to more than 1 year after operation had subtherapeutic levels in 10 to $50 \%$ of patients. One-half of the patients had more than 1 seizure a month. In temporal resection patients half of the recurrences were controllable, whereas patients with extratemporal resections had a greater likelihood $(96 \%)$ of persistent seizures and seizures became intractable in $74 \%$. The age at operation, the duration of epilepsy, and associated structural lesions did not influence the likelihood of persistent seizures or intractability after recurrence. (Wingkun EC, Awad IA et al. Natural history of recurrent seizures after resective surgery for epilepsy. Epilepsia Nov/Dec 1991; 32:851-856.) (Reprints: Dr. Awad, Department of Neurological Surgery, Cleveland Clinic Foundation, 9500 Euclid Ave., Cleveland, OH 44195.)

COMMENT. After the first seizure recurrence there appears to be a predictable trend of persistent seizures over the next 5 years. A decrease in seizure recurrence in the first 3 years after the first episode of relapse may reflect a more careful attention to antiepileptic drug therapy. Seizures recurring within 1 week postoperatively are not benign and have the same likelihood of subsequent intractability as other recurrent seizures in the first postoperative year. The outcome of resective surgery in extratemporal regions was less encouraging than temporal resections. If seizure recurrences are delayed until after the first postoperative year the outlook is better and seizures rarely become intractable. Changes in antiepileptic drugs, dosages or poor compliance may be important factors in recurrence of seizures. The maturing epileptogenicity at the scar of a previous resection does not appear to contribute to intractability of seizures but may be a factor in later recurrences.

Details of the anticonvulsant drug regimens used before and after operation were not provided and the comparative efficacy of phenytoin and carbamazepine in postoperative epilepsy would be of interest. A comparative study of the cognitive effects of phenytoin and carbamazepine in healthy young adults has shown no significant clinical differences between the 2 drugs. (Meador KJ et al. Comparative cognitive effects of carbamazepine and phenytoin in healthy adults. Neurology, 1991; $41: 1537$. 